\title{
A Toolbox of Potential Immune-Related Therapies for Inflammatory Cardiomyopathy
}

\author{
Ahmed Elsanhoury ${ }^{1,2} \cdot$ Carsten Tschöpe $^{1,2,3} \cdot$ Sophie Van Linthout ${ }^{1,2}$ (D)
}

Received: 5 March 2020 / Accepted: 7 May 2020 / Published online: 21 May 2020

(C) The Author(s) 2020

\begin{abstract}
Myocarditis is a multifactorial disorder, characterized by an inflammatory reaction in the myocardium, predominantly triggered by infectious agents, but also by antigen mimicry or autoimmunity in susceptible individuals. Unless spontaneously resolved, a chronic inflammatory course concludes with cardiac muscle dysfunction portrayed by ventricular dilatation, clinically termed inflammatory cardiomyopathy (Infl-CM). Treatment strategies aim to resolve chronic inflammation and preserve cardiac function. Beside standard heart failure treatments, which only play a supportive role in this condition, systemic immunosuppressants are used to diminish inflammatory cell function at the cost of noxious side effects. To date, the treatment protocols are expertbased without large clinical evidence. This review describes concept and contemporary strategies to alleviate myocardial inflammation and sheds light on potential inflammatory targets in an evidence-based order.
\end{abstract}

Keywords Inflammatory cardiomyopathy ·

Immunosuppression · Immunomodulation

$\begin{array}{ll}\text { Abbreviations } \\ \text { AZP } & \text { Azathioprine } \\ \text { B19V } & \text { Parvovirus B19 } \\ \text { CBD } & \text { Cannabidiol } \\ \text { CCL } & \text { CC-chemokine ligand } \\ \text { CsA } & \text { Cyclosporine A } \\ \text { COX } & \text { Cyclooxygenase } \\ \text { CVB3 } & \text { Coxsackievirus B3 } \\ \text { DCM } & \text { Dilated cardiomyopathy } \\ \text { EF } & \text { Ejection fraction } \\ \text { EMB } & \text { Endomyocardial biopsy } \\ \text { IFN } & \text { Interferon }\end{array}$

Associate Editor Saskia de Jager oversaw the review of this article

Sophie Van Linthout

Sophie.Van-linthout@charite.de

1 Berlin Institute of Health Center for Regenerative Therapies (BCRT), Charité - Universitätsmedizin Berlin, Campus Virchow Klinikum (CVK), Föhrerstrasse 15, 13353 Berlin, Germany

2 German Center for Cardiovascular Research (DZHK), Partner site Berlin, Berlin, Germany

3 Department of Cardiology, Charité - Universitätsmedizin Berlin, Campus Virchow Klinikum (CVK), Berlin, Germany

$\begin{array}{ll}\text { IL } & \text { Interleukin } \\ \text { Infl-CM } & \text { Inflammatory cardiomyopathy } \\ \text { LV } & \text { Left ventricular/left ventricle } \\ \text { mTOR } & \text { Mammalian target of rapamycin } \\ \text { MTX } & \text { Methotrexate } \\ \text { NF-kB } & \text { Nuclear factor-kB } \\ \text { NLRP3 } & \text { Nucleotide oligomerization domain (NOD)-, } \\ & \text { leucine-rich repeat (LRR)-, and pyrin domain- } \\ & \text { containing protein 3 } \\ \text { NSAIDs } & \text { Nonsteroidal anti-inflammatory drugs } \\ \text { RAAS } & \text { Renin angiotensin aldosterone system } \\ \text { ROS } & \text { Reactive oxygen species } \\ \text { RTX } & \text { Rituximab } \\ \text { TNF } & \text { Tumor necrosis factor } \\ T_{\text {reg }} & \text { Regulatory T cells }\end{array}$

\section{Introduction}

Inflammation, a complex biological response to injury, infection, and exposed autoantigens, sometimes stirs in the myocardium. Myocardial inflammation is characterized by nonspecific symptoms like chest pain, arrhythmias, and heart failure signs of non-ischemic origin [1, 2]. The inflammatory reaction is most frequently associated with viral infections including coxsackievirus B3 (CVB3), adenoviruses, and active parvovirus B19 (B19V), whereas trypanosomes, bacteria, toxic substances, and autoantigens are other frequent etiologies 
$[2,3]$. Myocarditis may spontaneously resolve without clinical footprints or remain active, instigating a chronic inflammatory course that culminates into inflammatory cardiomyopathy (Infl-CM), characterized by left ventricular (LV) dysfunction and heart failure or arrhythmias [1-4]. Cardiotropic microbes and cardiotoxins induce acute myocarditis via direct activation of the host immune system or via induction of myocyte necrosis and exposure of normally hidden antigens $[2,4]$. Although in the acute phase, cardiac inflammation can be detected by cardiac magnetic resonance imaging, endomyocardial biopsy (EMB) analysis is the only diagnostic tool capable of identifying the underlying etiology of cardiac inflammation, allowing quantification of immune cell subtypes and microbial nucleic acid [2, 5-7].

The associated inflammatory processes are diverse and complex, including microbial or nonmicrobial inducers (e.g., alarmins, extracellular matrix fragments, and selfproteins), which activate immune sensors (e.g., inflammasomes, Toll-like receptors) and several kinds of mediators including cytokines, chemokines (e.g., CCchemokine ligand (CCL)2 and CCL7), eicosanoids (e.g., prostaglandins), biogenic amines (e.g., histamine), and bioactive peptides (e.g., bradykinin) [8]. Being the major peripheral lymphatic reservoir of monocytes and filter of viruses, the spleen plays a major role in the development of Infl-CM. Monocytes from the spleen home to the heart (cardiosplenic axis), where they next contribute to tissue injury and cardiac remodeling [9-13].

Immunosuppressant agents like lympholytic, antiproliferative agents and proliferation signal inhibitors are seen as potential therapies for myocardial inflammation, frequently indicated as off-label treatments or used in the context of clinical studies. For the moment, most clinical studies in this arena are investigator-initiated, and there is a lack of sufficient information concerning the clinical value of individual immunosuppressive agents. Etiologybased treatment protocols are needed for which EMB analysis subclassifies the patients to different strata [8]. There is no available consensus in general, due to the lack of large patient registries and randomized controlled trials, yet this review aims to sketch the contemporary strategies promised to counteract myocardial inflammation and to minimize its impact on the myocardium. The order of available strategies is built on the degree of clinical and experimental evidence, starting with (I) global immunosuppressive strategies antagonizing cellular and humoral immunity, followed by strategies, which (II) systemically modulate the immune response, (III) antagonize key inflammatory components, (IV) reduce myocardial wall stress via mechanical unloading, and (V) decrease mimic peptides-driven anti-cardiac autoimmunity via antibiotic therapy (Table 1).
Strategy I: Global Immune System Suppression

Immunosuppressive therapies are used to counteract a broad range of deleterious conditions attributed to exaggerated or inappropriate immune responses responsible for acute or chronic inflammation. Agents of this pharmacological class are known prescription medications for autoimmune disorders like rheumatoid arthritis and for transplant rejection prevention [14]. Besides, immunosuppressive therapies are indicated to counteract unrestrained inflammation that persists in the absence of an inflammatory trigger. Leukocytes mediate inflammation and tissue damage through the release of lysosomal enzymes, toxic hydroxyl radicals, and chemotactic cytokines that further activate the inflammatory cascade and recruit collagen-producing cells [15]. The use of immunosuppression-based strategies in the context of myocarditis and Infl-CM is only recommended if active infection is ruled out via EMB-based molecular diagnostics [2]. Empirical immunosuppression treatment options for EMBproven microbial-negative myocardial inflammation, based on reported clinical evidence and expert-opinions, are discussed below and outlined in Fig. 1.

\section{Prednisolone, Azathioprine, and Cyclosporine}

Prednisolone is a synthetic corticosteroid, able to dramatically reduce inflammation irrespective of its origin, owning to its diverse suppressive effects on leukocytes and inflammatory mediators. Prednisolone inhibits leukocyte extravasation and reduces macrophage-phagocytic functions and production of TNF- $\alpha$, IFN- $\gamma$, IL-1, and IL-12. Besides, prednisolone has profound effects on eicosanoids via (1) inhibition of phospholipase A2, an enzyme responsible for the formation of arachidonic acid, the precursor of prostaglandins and leukotrienes, and (2) reduction in expression of cyclooxygenase enzyme (COX) isoform II, responsible for the synthesis of prostaglandins from arachidonic acid [16].

Azathioprine (AZP) is a prodrug metabolically activated to 6-mercaptopurine, which forms masquerade purine nucleotides, cytotoxic to activated lymphocytes. In addition, the prodrug is believed to induce antigen-specific tolerance by interfering with CD28 co-stimulatory signaling [16, 17].

Cyclosporine A (CsA) is a more potent, widely used immunosuppressant in organ transplantation and severe inflammatory disorders. This potent immunosuppressant forms a complex with a cytosolic immunophyllin, called cyclophilin, which upon formation inhibits calcineurin. Inhibition of calcineurin leads to a reduction in IL-2, IL-3, and IFN- $\gamma$ transcription and $\mathrm{T}$ cell activity $[16,18]$. The bioavailability of CsA varies among patients, which require individualized dose adjustment [16, 19]. CsA is known to increase the risk of lymphomas and other malignancies. Furthermore, it induces 
Table 1 Overview of available immune-related strategies suggested for the treatment of inflammatory cardiomyopathy

\begin{tabular}{|c|c|}
\hline Strategies & Working mechanism \\
\hline \multicolumn{2}{|c|}{ Strategy I: Global immune system suppression } \\
\hline $\begin{array}{l}\text { Prednisolone plus azathioprine } \\
\text { or cyclosporine A }\end{array}$ & $\begin{array}{l}\text { Prednisolone: leukocyte and eicosanoids suppression. Azathioprine: } \\
\text { depletion of activated lymphocytes and induction of antigen-specific } \\
\text { tolerance. Cyclosporine: calcineurin inhibition }\end{array}$ \\
\hline Mycophenolate mofetil & Selective T and B lymphocyte depletion \\
\hline Rituximab & Selective B lymphocyte depletion \\
\hline Methotrexate & Suppression of lymphocyte function \\
\hline Sirolimus & Inhibition of mTOR signaling \\
\hline \multicolumn{2}{|c|}{ Strategy II: Systemic modulation of the immune response } \\
\hline Intravenous immunoglobulins & $\begin{array}{l}\text { Buffering different pro-inflammatory responses. Aid in pathogen recog- } \\
\text { nition and clearance }\end{array}$ \\
\hline Interferon- $\beta$ & Regulation of cell-mediated immunity \\
\hline Autoantibody therapies & Depletion of autoantibodies \\
\hline Cannabidiol & $\begin{array}{l}\text { Attenuation of different immune-mediated cardiotoxic processes via un- } \\
\text { known mechanism(s) }\end{array}$ \\
\hline Cell-based therapies & Induction of/reestablishing immune tolerance \\
\hline \multicolumn{2}{|c|}{ Strategy III: Antagonizing key inflammatory components } \\
\hline Colchicine & Suppression of neutrophils and NLRP3 inflammasome signaling \\
\hline Anakinra or canakinumab & Antagonizing interleukin-1 \\
\hline Q-compounds & Antagonizing S100A8/S100A9 alarmins \\
\hline \multicolumn{2}{|c|}{ Strategy IV: Reducing myocardial wall stress via mechanical unloading } \\
\hline \multicolumn{2}{|c|}{ Strategy V: Decreasing mimic peptides-driven anti-cardiac autoimmunity via antibiotic therapy } \\
\hline
\end{tabular}

mTOR, mammalian target of rapamycin; NLRP3, nucleotide oligomerization domain (NOD)-, leucine-rich repeat (LRR)- and pyrin domain-containing protein 3 irreversible renal impairment, mandating close monitoring of renal function.
In lymphocytic myocarditis, prednisolone alone or in combination with AZP and/or CsA is the most frequently used
Fig. 1 Empirical

immunosuppression treatment options for endomyocardial biopsy-proven microbial-negative myocardial inflammation, based on reported clinical evidence and expert-opinions. Red gradient indicates higher risk of

immunosuppression-associated side effects and poorer clinical experience. Different immunosuppressive combinations are clinically relevant in cases of drug-specific contraindications and/or severe side effects. AZP, azathioprine; MTX, methotrexate; RTX, rituximab; MMF, mycophenolate mofetil. *AZP is not recommended in cases with chronic liver disease. **Singlecenter experience, used in patients developing nephrotoxicity in response to CsA. *** Used in cases with EMB-proven persistent CD20+ B lymphocyte infiltrates

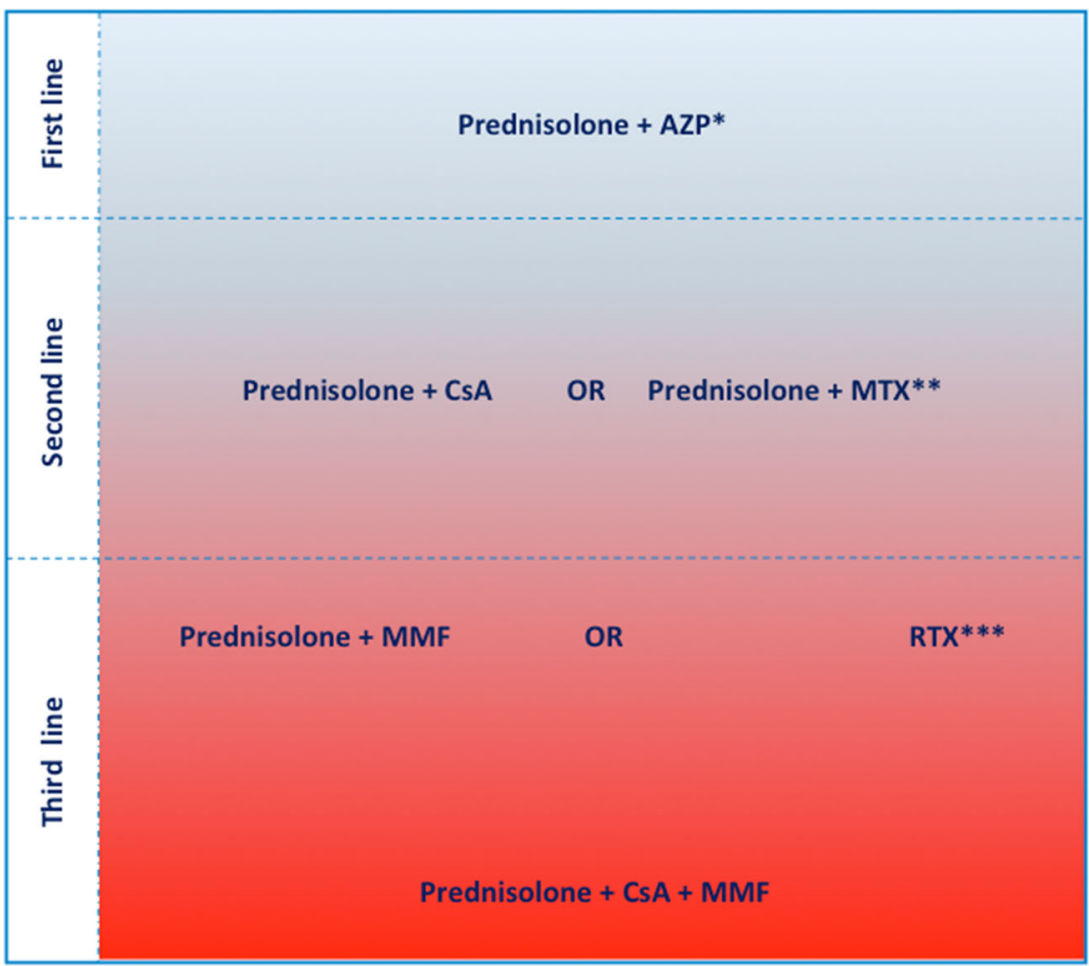


immunosuppressive regimens. The outcomes of prednisolone/ AZP combination therapy in virus-negative Infl-CM are largely favorable. A large retrospective controlled study including 90 patients per group (propensity score matched) demonstrated significant improvements in LV ejection fraction (EF) and heart transplantation-free survival in the group treated with prednisolone/AZP combination compared with the control group treated with standard heart failure medications [20]. The TIMIC study, which employed a prospective randomized, double-blind, placebo-controlled design demonstrated the efficacy of the combination therapy in virus-negative Infl-CM patients [21]. Favorable long-term outcomes were demonstrated in similar patients selected according to the ESC EMB-based diagnostic criteria [22]. Recently, Tschöpe et al. showed a therapeutic value for prednisolone/AZP combination in B19V DNA-positive myocarditis patients. The regimen downsized inflammation, yet, left B19V DNA copy number unaffected [23]. CsA combined with prednisolone is sometimes used to counteract severe cardiac muscle diseases including giant cell myocarditis, eosinophilic myocarditis, and cardiac sarcoidosis [7, 24]. CsA/prednisolone combination therapy was associated with EMB-proven improvement of myocarditis in infants with Infl-CM [25]. Cyclophilin, the pharmacological target of cyclosporine $\mathrm{A}$, was found to be enhanced in EMB samples from Infl-CM patients [26] and to contribute to inflammation in a murine CVB3-induced myocarditis model, suggesting CsA as potential therapy [27]. Theoretically, tacrolimus, another calcineurin inhibitor, could be of therapeutic value. However, its use in myocarditis and Infl-CM patients is restrained, since the drug is alleged to contribute to cardiac toxicity, driving different forms of cardiomyopathies [28-31].

\section{Mycophenolate Mofetil}

Mycophenolate mofetil (MMF), a prodrug of mycophenolic acid, which has immunosuppressive and anti-proliferative properties, is the drug of choice in solid organ transplant patients with refractory rejection $[16,32]$. The active acid inhibits de novo synthesis of purines, leading to a nearly selective inhibition of DNA replication in T and B lymphocytes [16, 32-34]. Besides, MMF impedes leukocyte adhesion [16, 35]. Owing to multiple mechanisms of action, MMF abrogates both cellular and humoral immunity. In an experimental autoimmune myocarditis murine model, MMF has been shown to interfere with the disease development [36]. Another murine study observed an improvement in CVB3induced myocarditis in MMF-treated mice [37]. De Luca et al. assessed the efficacy of MMF in a small cohort of virus-negative myocarditis patients who were intolerant/ refractory to AZP. A 6-month treatment course improved cardiac function and reduced the mean $\mathrm{LV}$ end diastolic diameter together with the circulating markers troponin T and NT-
proBNP [38]. A recent case report has presented the efficacy of triple immunosuppressive therapy comprising prednisolone/CsA and MMF in a cardiogenic shock case with recurrent giant cell myocarditis. The addition of MMF to the dual immunosuppressive therapy abrogated myocardial inflammation and allowed steroid dose reduction and full recovery [39]. In another case report, Tschöpe et al. revealed an increased presence of CD20+ B lymphocytes in lymphocytic myocarditis patients refractory to combined immunosuppressive therapy [40]. This phenotype is a good example for the requirement of personalized treatment and could be targeted with MMF. Whether MMF treatment is efficient to treat those patients has not been investigated so far.

\section{Rituximab}

Rituximab (RTX) is a chimeric monoclonal antibody that binds to the $\mathrm{CD} 20$ protein on the $\mathrm{B}$ lymphocyte surface, mediating specific depletion of CD20+ B lymphocytes via apoptosis stimulation and complement-dependent/cellular-mediated cytotoxicity. Clinically, RTX is indicated for B nonHodgkin's lymphomas, chronic lymphocytic leukemia, and Wegener's disease $[16,41,42]$. CD20+ B lymphocytes contribute to myocardial damage directly as well as via amplifying the effector functions of $\mathrm{T}$ lymphocytes and monocytes [43-47]. Notably, CD20+ B lymphocytes are resilient against steroid-based therapies $[40,48]$. A case series described a subset of Infl-CM patients, who were refractory to combined steroid-based immunosuppression. The specific patients showed CD20+ B lymphocytic infiltrates in the EMB samples, which resolved upon RTX treatment, mirrored with clinical improvement [40].

\section{Methotrexate}

Methotrexate (MTX) is a disease modifying antirheumatic drug, used in $50-70 \%$ of patients with rheumatoid arthritis. At low doses, MTX interferes with thymidine and adenosine metabolism leading to an elevation in the extracellular adenosine level. The latter is a potent inhibitor of inflammation via suppressing antigen-dependent immune cell activation and chemotaxis. Besides, MTX is an antimetabolite that inhibits dihydrofolate reductase, a critical enzyme in the de novo synthesis of thymidine, leading to a block in DNA replication and subsequent repression of lymphocytes formation and function $[16,49]$. A prospective study by Choi et al. reported that MTX reduces all-cause mortality of rheumatoid arthritis patients, mainly by reducing cardiovascular deaths by $70 \%$ [50], though the cardiovascular-protective effect was not confirmed in atherosclerosis patients [51]. Yet, the pharmacological mechanism of MTX remains relevant and is worth investigating in myocarditis. Few experts use MTX in steroidscombined immunosuppression regimens for patients 
intolerant for AZP or CsA (Fig. 1). So far, there are no published clinical data regarding the efficacy and safety of this regimen.

\section{Sirolimus}

Sirolimus, also called rapamycin, is a relatively new immunosuppressant with anti-proliferative effects due to its ability to inhibit a key protein in cell proliferation, called mammalian target of rapamycin (mTOR), and ultimately inhibits IL-2mediated $\mathrm{T}$ (mainly) and B lymphocyte activation [16, 52]. Moreover, sirolimus was shown to reduce inflammation via the reduction of IL-6 formation [53]. Thanks to its antiproliferative properties, sirolimus-eluting coronary stents are used in clinical practice to prevent restenosis $[54,55]$. mTOR was shown to be involved in cardiac inflammation via NF-kB signaling, whereas its inhibition may modify cardiac inflammation and hypertrophy [56]. Several evidences supporting the potential of mTOR-inhibition as a strategy against cardiomyopathy were reviewed by Kuschwaha et al. [57]. Recently, an in silico drug repositioning study [58] has identified sirolimus as a potential therapeutic option for Infl-CM, based on a systemic comparison of drug-induced gene expression profiles from twelve patients. However, the computational approach was limited by the very small sample size. In cell culture experiments, sirolimus has been shown to promote CVB3-induced cytopathic effects and apoptosis [59]. This highlights that the use of sirolimus in virus-positive myocarditis could be hazardous. Currently, there are no sufficient clinical data advocating the off-label use of sirolimus in myocardial inflammation.

\section{Strategy II: Systemic Modulation of the Immune Response}

An alternative approach to systemic immune suppression is the regulatory adjustment of specific immune and inflammatory responses by the action of an immunomodulator. In this section, the use of different immunomodulatory strategies in Infl-CM is overviewed.

\section{Intravenous Immunoglobulins}

Human intravenous immunoglobulins (IVIGs) are unspecific polyclonal antibodies extracted from pooled human sera. IVIGs have normalizing effects on the host immune system mediated via inhibiting pro-inflammatory cytokines, inducing anti-inflammatory cytokines, expanding regulatory $\mathrm{T}$ cells $\left(\mathrm{T}_{\text {reg }}\right)$, and blocking pathogenic antibodies via idiotypic-antiidiotypic interactions and $\mathrm{Fc}$ receptor binding. In addition, IVIGs therapy aids to clear pathogens via pathogen opsonization, complement, and effector cell activation
[60-63]. IVIGs provide an immunomodulatory therapeutic option for several autoimmune and inflammatory disorders [64-66]. The therapeutic utility of IVIGs in Infl-CM is controversial. On the one hand, the IMAC (Intervention in Myocarditis and Acute Cardiomyopathy) study could not proof the benefit of IVIGs treatment in acute myocarditis patients [67], whereas it seems particularly effective for the treatment of neonates with fulminant enteroviral infection [68]. Furthermore, small-scale studies and patient registries showed positive treatment outcomes including clearance of inflammation even in virus-associated myocarditis. Yet virus eradication was imperfect [69-73]. A double-blind placebo-controlled study on B19V cardiomyopathy patients, intended to disentangle the current knowledge, has been completed [NTC00892112].

\section{Interferon-ß}

IFN- $\beta$ is an endogenous cytokine carrying immunomodulatory and antiviral functions attributed to augmenting antigen presentation and immune cell activation $[74,75]$. The clinical value of IFN- $\beta$ in Infl-CM has been related to viral etiologies, in particular CVB3 and adenovirus, where immunosuppression is contraindicated [5]. Clinical studies employing 6month IFN- $\beta$ regimen have been associated with myocardial CVB3 and adenovirus clearance, reduced myocardial CD3+ T cell infiltration, and clinical improvement $[76,77]$.

\section{Autoantibody Therapies}

Immune adsorption, classically used to remove autoantibodies from blood, has also been investigated in DCM patients with cardiodepressant autoantibodies [72, 78-80]. Immune adsorption therapy is under current investigation in clinical studies and so far considered for both virus-positive/virus-negative patients refractory to systemic immunosuppression. Alternatively, autoantibodies can be neutralized through the intravenous application of small soluble molecules, including peptides or aptamers [81].

\section{Cell-Based Therapies}

$\mathrm{T}_{\text {reg }}$ are a subpopulation of CD4+ cells that function to maintain immune tolerance and prevent autoimmunity. $T_{\text {reg }}$ cells have been shown to be dysregulated and in imbalance with pro-inflammatory $\mathrm{Th}_{17}$ cells, in myocarditis and autoimmune DCM [82-85]. Reestablishing the quality and the quantity of the $T_{\text {reg }}$ population via direct application into the circulation has been shown to be a successful therapeutic strategy in a murine model of CVB3-induced myocarditis $[82,85,86]$.

Mesenchymal stromal cells (MSCs) are plastic-adherent cells that can be isolated from bone marrow and adipose and other body tissues. MSCs are immunoprivileged, exert 
immunomodulatory properties [87], and are able to home to sites of inflammation, offering therapeutic potential for myocarditis $[88,89]$. In a murine CVB3-induced myocarditis model, MSCs have been described to increase circulating $\mathrm{T}_{\text {reg }}$ cells [90], modulate monocyte trafficking [9], and inhibit activation of nucleotide oligomerization domain (NOD)-, leucine-rich repeat (LRR)- and pyrin domain containing protein 3 (NLRP3) [91] among other cardioprotective effects. Similar to MSCs, cardiac stromal cells derived from endomyocardial biopsies exert immunomodulatory [92] and cardioprotective [93] effects. Their potential to increase $T_{\text {reg }}$ cells, to decline cardiac mononuclear cell activity, and to improve LV function has been shown in an experimental model of CVB3-induced myocarditis [94].

\section{Cannabidiol}

Cannabidiol (CBD) is a non-psychoactive cannabis extract, currently under investigation in numerous indications owing to the anti-inflammatory, antioxidant, and cytoprotective effects, and it exerts independent of classical $G$ protein coupled cannabinoid receptors activation $[95,96]$. Addressing cardiomyopathies, CBD has been shown to offer cardioprotective effects in diabetic cardiomyopathy and in doxorubicininduced cardiomyopathy [96, 97]. In a chronic autoimmune myocarditis murine model, Lee et al. have demonstrated that long-term CBD treatment could attenuate $\mathrm{T}$ cell responses, oxidative stress, and fibrosis [98]. The anti-inflammatory/immunomodulatory effects of CBD are likely to be beneficial in other forms of myocarditis.

\section{Strategy III: Antagonizing Key Inflammatory Components}

Nonsteroidal anti-inflammatory drugs (NSAIDs) are designed to abrogate the synthesis of prostaglandins - a class of eicosanoids - by inhibiting the COX isoforms, responsible for the synthesis of prostaglandins from arachidonic acid. Yet the use of NSAIDs is of limited long-term utility in cardiovascular disorders since they also mediate salt and water retention, and thrombus formation (COX-II inhibitors) worsening heart failure, and therefore not recommended for myocarditis treatment $[99,100]$. The present paragraph resumes emerging anti-inflammatory agents for myocarditis or InflCM (Fig. 2).

\section{Antagonizing Neutrophils and NLRP3/IL-1ß Pathway via Colchicine}

Colchicine is an anti-inflammatory alkaloid, traditionally used for treatment of and prophylaxis against acute gouty arthritis [101]. The alkaloid interferes with microtubule polymerization, which disrupts the cytoskeleton and arrests mitosis in all rapidly dividing cells. The impact of colchicine on the cytoskeleton critically affects neutrophils functions including chemotaxis, adhesion, and motility. In addition, colchicine reduces superoxide production and inhibits the NLRP3 inflammasome and IL-1 $\beta$ formation (Fig. 2). Together with innate immunity modulation, colchicine exerts anti-inflammatory, anti-fibrotic, and endothelial protective effects [101-103]. The NLRP3 inflammasome, which can be activated via pathogen structures or sterile stimuli, has been identified as inflammatory mediator in myocarditis [91, 104, 105], escorting poor long-term outcomes [106]. In a murine myocarditis model, colchicine abrogated myocardial inflammation, linked with splenic NLRP3 reduction [107]. In clinical studies, colchicine demonstrated favorable treatment outcomes in coronary artery disease [108], pericarditis [109-111], and post-pericardiotomy syndrome [112]. Pericarditis, which is frequently associated with myocarditis, is often treated with colchicine under the recommendation of the most recent ESC guidelines [113]. Since the inflammatory processes and the role of cardiotropic viruses do not differ, in the most cases, between peri- and myocarditis, colchicine is suggested to be effective in myocarditis/Infl-CM, too. In line, a case report has indicated the efficacy of colchicine to treat patients with myocarditis [114].

\section{Antagonizing IL-1 via Anakinra and Canakinumab}

NLRP3 inflammasome activation culminates - through several steps - into the proteolytic cleavage of pro-IL-1 and pro-IL-18 to the active cytokine forms IL-1ß and IL18 [115-117]. Murine model studies described the pivotal role of IL-1ß in the pathogenesis of autoimmune and viral myocarditis, highlighting the therapeutic potential of IL-1Bblocking agents $[118,119]$. Anakinra is a competitive IL-1 receptor antagonist (Fig. 2), approved for rheumatoid arthritis patients, who are not responding to conventional disease modifying agents [120]. Rheumatic disease is commonly associated with extra-articular cardiovascular manifestation. There was early evidence that anakinra treatment can improve cardiac function in acute rheumatoid arthritis patients [121]. Randomized controlled studies further demonstrated the advantageous outcomes of anakinra treatment in serious cardiac conditions including acute myocardial infarction [122], acute decompensated heart failure [123], heart failure with preserved ejection fraction [124], and pericarditis [125]. In two case reports, Cavali et al. described dramatic improvement of fulminant myocarditis derived-cardiogenic shock shortly after initiation of anakinra [126]. At the moment the superiority of anakinra to standard heart failure treatment in acute myocarditis is being investigated in a triple-blind randomized clinical trial [ARAMIS; NCT03018834]. An alternative mechanism is 


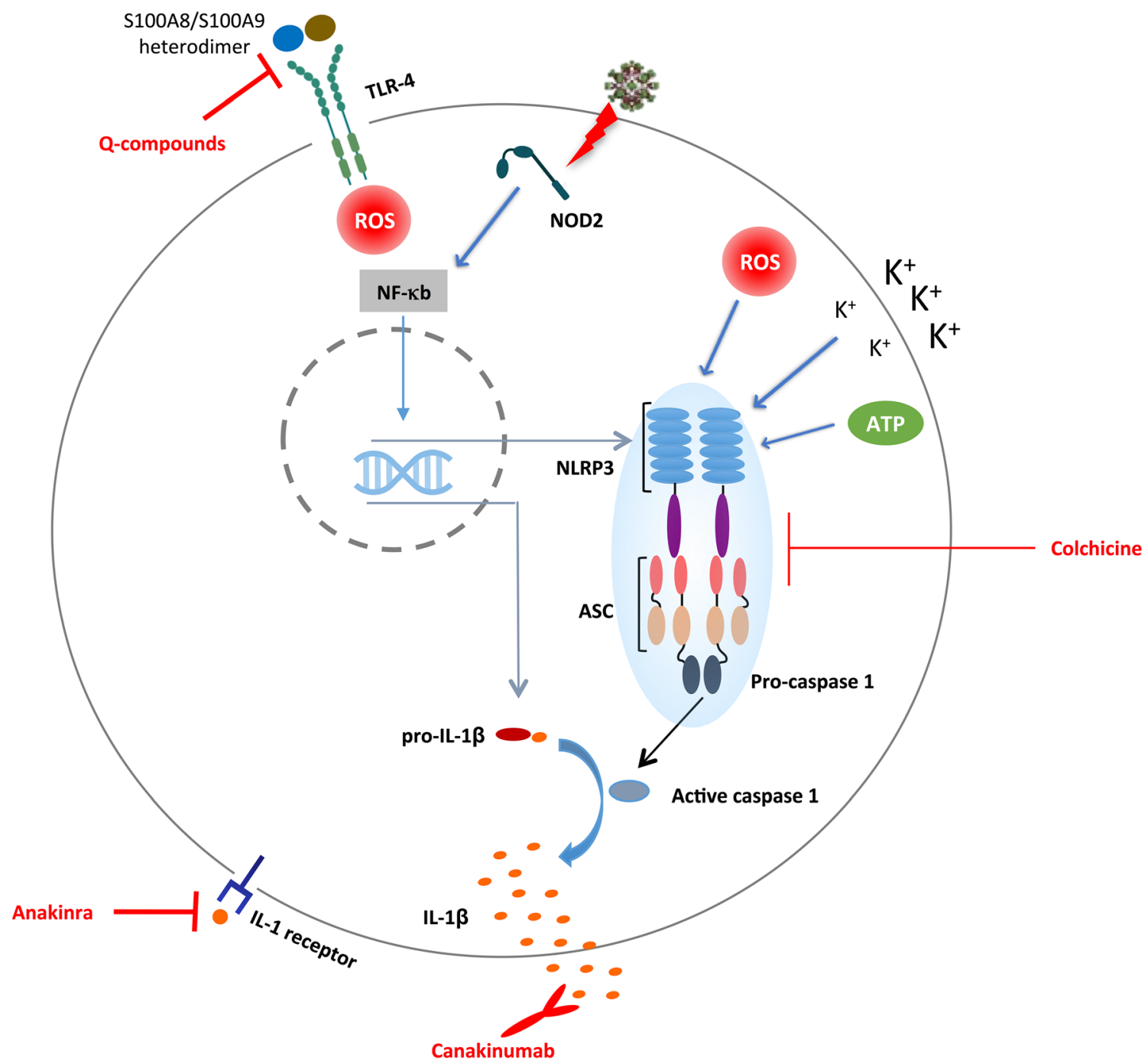

Fig. 2 Strategies for antagonizing NLRP3 pathway myocarditis-related key inflammatory components. (1) Upon binding of the S100A8/S1009 heterodimer to the Toll-like receptor 4 (TLR 4) or receptor for advanced glycation end product (RAGE) on an innate immune cell, reactive oxygen species (ROS) are generated in the cytosol, which leads to the activation and translocation of the nuclear factor kappa B $(\mathrm{NF}-\mathrm{kB})$ to the nucleus. Alternatively, viral single-stranded RNA, as by coxsackievirus B3 (CVB3), can activate the intracellular receptor nucleotide-binding oligomerization domain-containing protein 2 (NOD-2), which also activates $\mathrm{NF}-\mathrm{kB}$. The latter acts as transcription factor that stimulates the mRNA expression of the nucleotide the oligomerization domain-containing,

offered by canakinumab (Fig. 2), a monoclonal antibody that binds and neutralizes IL-1ß [16]. The CANTOS trial, which employed canakinumab as an anti-inflammatory strategy in atherosclerotic patients, reported lower rate of cardiovascular events and circulating levels of IL-1ß, IL-6, together with high-sensitivity C-reactive protein [127]. To date, the efficacy of canakinumab in myocarditis treatment is not reported. In contrast to transplant-related immunosuppressant agents, cytokine inhibitors portray a rapid onset of action and a remarkably higher safety profile. Yet, blocking IL-1 1 is still associated with a high risk of infection [127].

leucine-rich repeat-containing, and pyrin domain-containing protein (NLRP3) inflammasome and of pro-IL-1ß. (2) Upon activation by K+ efflux, ATP, ROS, and other damage-associated molecular patterns, NLRP3 polymerizes with the adaptor protein ASC and caspase 1. Subsequently, caspase 1 cleaves pro-IL- $1 \beta$ to its active form: IL-1ß. (3) The active cytokine IL-1ß is secreted to the extracellular space, where it binds to its receptor and induces autocrine and paracrine signaling on other immune cells. Q-compounds block step (1) upstream; colchicine blocks step (2); canakinumab and anakinra block step (3) via different mechanisms

\section{Antagonizing S100A8/S100A9 Alarmins via Q- Compounds}

S100A8 and S100A9 alarmins are pro-inflammatory proteins secreted by phagocytes during inflammation [128]. In the presence of calcium, S100A8 and S100A9 form heterodimers that bind to Toll-like receptor (TLR) 4 and receptor for advanced glycation end products (RAGE), expressed on the surface of peripheral blood mononuclear cells. TLR 4 and RAGE stimulate the production of reactive oxygen species that act as secondary messengers to activate the transcription factor 
NF- $\kappa \mathrm{B}$, which in turn - in the presence of ATP_-leads to the expression of NLRP3 and pro-IL1ß. The latter is further cleaved via caspase-1 resulting in the secretion of the active cytokine IL1ß [129-131] (Fig. 2). S100A8 and S100A9 have been shown to correlate with different cardiovascular diseases including myocardial infarction and myocarditis [80, 132, 133]. In CVB3-positive myocarditis patients, the expression of S100A8 and S100A9 in EMB specimens was associated with bad prognosis [134]. Experimental immunomodulatory compounds of the quinoline-3-carboxamides family, referred to as Q-compounds, offer a novel pharmacological approach to treat autoimmune/inflammatory diseases like systemic lupus erythematosus via blocking the binding of S100A9 to TLR 4 [135]. Treating specific Infl-CM patients with Qcompounds based on S100A8/S100A9 serum levels seems to be a promising target-directed strategy, worth evaluating in clinical studies [8].

\section{Strategy IV: Reducing Myocardial Wall Stress via Mechanical Unloading}

Myocardial inflammation may lead to contractile dysfunction and provoke hemodynamic compromise and an increase in wall stress [136]. The latter in turn can activate mechano-transduction pathways, which forces a myocardial inflammatory status [137]. Local and systemic activation of the renin angiotensin aldosterone system (RAAS) in overloaded hearts contributes to myocardial inflammation and remodeling $[137,138]$. Interestingly, $\mathrm{T}$ lymphocytes and macrophages have been shown to respond to mechanical stress $[139,140]$. Mechanically induced T lymphocyte activation further drives extracellular matrix deposition and fibrosis [139]. In macrophages, mechanical stress can advance a pro-fibrogenic phenotype [140].

Axial flow pumps like Impella 2.5, CP, and 5.0 devices allow mechanical unloading of the LV, which does not only reduce myocardial work, energy expenditure, and oxygen demand, but also reduce wall stress [136]. In combination with prednisolone/AZP immunosuppression, prolonged Impella (PROPELLA) support performed via an axillary artery implanted impella 5.0 for up to 39 days has been shown to abrogate myocardial expression of S100A8 and S100A9 alarmins, adhesion molecules, and integrins, in line with reduction in immune cell infiltration, enhancing recovery [141]. The effects were abrogated after explantation of the Impella, despite continuation of immunosuppressive therapy, suggesting an unloading-dependent mechanism. The PROPELLA concept and mode-of-action was further confirmed in a HIV-positive patient in cardiogenic shock due to an EMBproven viral-negative fulminant myocarditis, by which PROPELLA was performed in the absence of immunosuppressive therapy due to preexisting AIDS [141]. Accumulating evidence advocates mechanical unloading as valuable treatment for compromised myocarditis patients. Large-scale clinical studies are necessary to further confirm the disease-modifying actions of PROPELLA beyond mechanical circulatory support.

\section{Strategy V: Decreasing Mimic Peptides-Driven Anti-Cardiac Autoimmunity via Antibiotic Therapy}

Environmental and genetic factors are considered critical determinants of myocarditis pathogenesis [142]. The exposure of normally hidden myocardial antigens following infections can predispose myocarditis in genetically susceptible individuals [143]. Gut microbiota can prime $\mathrm{T}_{\mathrm{H}}$ cells against bacterial antigens that mimic cardiac antigens like myosin heavy chain. Following subclinical ischemia or infection that allow antigen exposure, cross-reactive microbiota-driven $\mathrm{T}_{\mathrm{H}}$ cells can promote myocardial damage. [144-146]. Gil-Cruz et al. [146] showed that broad-spectrum antibiotic treatment could dampen $\mathrm{T}_{\mathrm{H}}$ cell-mediated cardiac inflammatory responses, trigged by commensal Bacteroides theca mimic peptides. This might also be of relevance in the context of virus-induced or virusassociated Infl-CM and needs further investigation.

\section{Discussion}

Systemic immunosuppression is the most potent strategy to defeat myocardial inflammation. Theoretically, any combination of immunosuppressant agents that harbor no hazardous interactions can be considered for Infl-CM treatment. The choice of whether to administer immunosuppressive agents should be seen as personalized practice, based on the whole clinical picture including other comorbidities, type of immune infiltrates, and microbes detected in the myocardium. Every regimen has to be weighed against the risk of infection, including reactivation of latent cardiac microbial infections, outburst of present cardiac virulent infections, and novel infections. Corticosteroids are found almost indispensable in every immunosuppressive combination, attributed to their chief anti-inflammatory activity. The dose of steroids required to produce an anti-inflammatory action is lower, compared with the immunosuppressive dose $[16,147]$. Combining prednisolone with potent immunosuppressant agents allows steroid dose reduction while preserving the anti-inflammatory mechanism. This approach shields prone patients like diabetics against steroid adverse effects. Clinical experience with the individual immunosuppressive agents is a major factor in the selection of the regimen by the treating physicians, which favors corticosteroids to a large extend. An immunosuppressive regimen should be initiated at low dose with up titration and may be stepped up to include different classes of 
immunosuppressant agents (Fig. 1), while through blood levels and common side effects need to be real-time monitored. The treatment protocols need to be shaped and synchronously refined based on the treatment associated benefits and risks aiming to achieve the best therapeutic outcome. More specific strategies meant to block specific myocarditis-related inflammatory mechanisms are currently under investigation yet barely used clinically against myocarditis due to the very limited availability of safety and efficacy data. Colchicine, the relatively cheap old-timer drug, can be an alternative for patients intolerant to steroids. Antagonizing the key inflammatory cytokine IL- $1 ß$ is a more specific, relatively safe and rapid onset treatment approach. However, combining systemic immunosuppression agents like corticosteroids or MMF with cytokine blocking agents is limited by a high risk of infection. Colchicine as steroid-free option, not associated with global immune suppression side effects, is safer to combine with IL$1 \beta$ antagonists. Moreover, the combination provides a dual approach to block the NLRP3 inflammasome pathway. Autoantibody therapies are considered the last line option for Infl-CM treatment, while mechanical unloading approaches seem favorable in acute cardiogenic shock cases.

\section{Conclusion}

Despite the advancements in basic and clinical research, Infl$\mathrm{CM}$ remains to be an unmet medical need. The different immunosuppressive and anti-inflammatory strategies described in this review are based on single-center experiences and small-scale studies. Treatment of myocarditis is mainly based on the off-label use of major systemic immunosuppressive agents, associated with numerous side effects. Several therapeutic targets revealed by basic research still need to be translated to the bedside. Large-scale randomized placebocontrolled clinical studies are of absolute necessity to designate safe and effective treatments.

Acknowledgements Open Access funding provided by Projekt DEAL.

\section{Compliance with Ethical Standards}

Conflict of Interest The authors declare that they have no conflict of interest.

Ethical Approval This article does not contain any studies with human participants or animals performed by any of the authors.

Open Access This article is licensed under a Creative Commons Attribution 4.0 International License, which permits use, sharing, adaptation, distribution and reproduction in any medium or format, as long as you give appropriate credit to the original author(s) and the source, provide a link to the Creative Commons licence, and indicate if changes were made. The images or other third party material in this article are included in the article's Creative Commons licence, unless indicated otherwise in a credit line to the material. If material is not included in the article's Creative Commons licence and your intended use is not permitted by statutory regulation or exceeds the permitted use, you will need to obtain permission directly from the copyright holder. To view a copy of this licence, visit http://creativecommons.org/licenses/by/4.0/.

\section{References}

1. Ammirati, E., et al. (2018). Clinical presentation and outcome in a contemporary cohort of patients with acute myocarditis. Circulation, 138(11), 1088-1099.

2. Caforio, A. L., et al. (2013). Current state of knowledge on aetiology, diagnosis, management, and therapy of myocarditis: A position statement of the European Society of Cardiology Working Group on Myocardial and Pericardial Diseases. European Heart Journal, 34(33), 2636-2648 2648a-2648d.

3. Cooper Jr., L. T. (2009). Myocarditis. The New England Journal of Medicine, 360(15), 1526-1538.

4. Kindermann, I., et al. (2012). Update on myocarditis. Journal of the American College of Cardiology, 59(9), 779-792.

5. Van Linthout, S., \& Tschope, C. (2018). Viral myocarditis: A prime example for endomyocardial biopsy-guided diagnosis and therapy. Current Opinion in Cardiology, 33(3), 325-333.

6. Tschope, C., Kherad, B., \& Schultheiss, H. P. (2015). How to perform an endomyocardial biopsy? Türk Kardiyoloji Derneği Arşivi, 43(6), 572-575.

7. Tschope, C., et al. (2019). Management of myocarditis-related cardiomyopathy in adults. Circulation Research, 124(11), 15681583.

8. Van Linthout, S., \& Tschope, C. (2019). The quest for antiinflammatory and immunomodulatory strategies in heart failure. Clinical Pharmacology and Therapeutics, 106(6), 1198-1208.

9. Miteva, K., et al. (2017). Mesenchymal stromal cells modulate monocytes trafficking in coxsackievirus B3-induced myocarditis. Stem Cells Translational Medicine, 6(4), 1249-1261.

10. Swirski, F. K., et al. (2009). Identification of splenic reservoir monocytes and their deployment to inflammatory sites. Science, 325(5940), 612-616.

11. Nahrendorf, M., Pittet, M. J., \& Swirski, F. K. (2010). Monocytes: Protagonists of infarct inflammation and repair after myocardial infarction. Circulation, 121(22), 2437-2445.

12. Leuschner, F., et al. (2015). Silencing of CCR2 in myocarditis. European Heart Journal, 36(23), 1478-1488.

13. Klingel, K., et al. (1996). Pathogenesis of murine enterovirus myocarditis: Virus dissemination and immune cell targets. Journal of Virology, 70(12), 8888-8895.

14. Holt, C. D. (2017). Overview of immunosuppressive therapy in solid organ transplantation. Anesthesiology Clinics, 35(3), 365380.

15. Mack, M. (2018). Inflammation and fibrosis. Matrix Biology, 6869, 106-121.

16. Katzung, B. G. (2018). Basic \& clinical pharmacology (14th ed.). New York: McGraw-Hill.

17. Maltzman, J. S., \& Koretzky, G. A. (2003). Azathioprine: Old drug, new actions. The Journal of Clinical Investigation, 111(8), $1122-1124$.

18. Youn, T. J., et al. (2002). Effects of the calcineurin dependent signaling pathway inhibition by cyclosporin a on early and late cardiac remodeling following myocardial infarction. European Journal of Heart Failure, 4(6), 713-718.

19. Kokuhu, T., et al. (2013). Dose adjustment strategy of cyclosporine A in renal transplant patients: Evaluation of anthropometric 
parameters for dose adjustment and $\mathrm{C} 0$ vs. $\mathrm{C} 2$ monitoring in Japan, 2001-2010. Int J Med Sci, 10(12), 1665-1673.

20. Merken, J., et al. (2018). Immunosuppressive therapy improves both short- and long-term prognosis in patients with virusnegative nonfulminant inflammatory cardiomyopathy. Circulation. Heart Failure, 11(2), e004228.

21. Frustaci, A., Russo, M. A., \& Chimenti, C. (2009). Randomized study on the efficacy of immunosuppressive therapy in patients with virus-negative inflammatory cardiomyopathy: The TIMIC study. European Heart Journal, 30(16), 1995-2002.

22. Escher, F., et al. (2016). Long-term outcome of patients with virus-negative chronic myocarditis or inflammatory cardiomyopathy after immunosuppressive therapy. Clinical Research in Cardiology, 105(12), 1011-1020.

23. Tschope, C., et al. (2019). Immunosuppression in inflammatory cardiomyopathy and parvovirus B19 persistence. European Journal of Heart Failure, 21(11), 1468-1469.

24. Cooper Jr., L. T., et al. (2008). Usefulness of immunosuppression for giant cell myocarditis. The American Journal of Cardiology, 102(11), 1535-1539.

25. Kleinert, S., et al. (1997). Myocarditis in children with dilated cardiomyopathy: Incidence and outcome after dual therapy immunosuppression. The Journal of Heart and Lung Transplantation, 16(12), 1248-1254.

26. Seizer, P., et al. (2013). EMMPRIN and its ligand cyclophilin a as novel diagnostic markers in inflammatory cardiomyopathy. International Journal of Cardiology, 163(3), 299-304.

27. Seizer, P., et al. (2012). Cyclophilin a affects inflammation, virus elimination and myocardial fibrosis in coxsackievirus B3-induced myocarditis. Journal of Molecular and Cellular Cardiology, 53(1), 6-14.

28. Pappas, P. A., et al. (2000). Sirolimus in pediatric gastrointestinal transplantation: The use of sirolimus for pediatric transplant patients with tacrolimus-related cardiomyopathy. Pediatric Transplantation, 4(1), 45-49.

29. McLeod, J., et al. (2017). Tacrolimus-associated dilated cardiomyopathy in adult patient after orthotopic liver transplant. Journal of Investigative Medicine High Impact Case Reports, 5(2), 2324709617706087.

30. Noda, K., et al. (2017). Tacrolimus-induced hypertrophic cardiomyopathy in a patient with dermatomyositis. Rheumatology (Oxford), 56(11), 2037-2038.

31. Bowman, L. J., et al. (2015). Tacrolimus-induced cardiomyopathy in an adult renal transplant recipient. Pharmacotherapy, 35(12), $1109-1116$.

32. Allison, A. C., \& Eugui, E. M. (2000). Mycophenolate mofetil and its mechanisms of action. Immunopharmacology, 47(2-3), 85118.

33. Egidio, D., et al. (1994). De novo purine nucleotide synthesis in total peripheral blood lymphocytes from patients with B-chronic lymphocytic leukemia (B-CLL). Biochemical Society Transactions, 22(2), 241S.

34. Parnham, M. (2011). Principles of immunopharmacology (3rd ed.). Basel: Springer.

35. Blaheta, R. A., et al. (1998). Inhibition of endothelial receptor expression and of T-cell ligand activity by mycophenolate mofetil. Transplant Immunology, 6(4), 251-259.

36. Kamiyoshi, Y., et al. (2005). Mycophenolate mofetil prevents the development of experimental autoimmune myocarditis. Journal of Molecular and Cellular Cardiology, 39(3), 467-477.

37. Padalko, E., et al. (2003). Mycophenolate mofetil inhibits the development of Coxsackie B3-virus-induced myocarditis in mice. BMC Microbiology, 3, 25.

38. De Luca, G., et al. (2019). Efficacy and safety of mycophenolate mofetil in patients with virus-negative lymphocytic myocarditis: A prospective cohort study. Journal of Autoimmunity, 102330.
39. Akita, T., et al. (2019). Successful triple combination immunosuppressive therapy with prednisolone, cyclosporine, and Mycophenolate Mofetil to treat recurrent giant cell myocarditis. Internal Medicine, 58(14), 2035-2039.

40. Tschope, C., et al. (2019). Targeting CD20+ B-lymphocytes in inflammatory dilated cardiomyopathy with rituximab improves clinical course: A case series. Eur Heart J Case Rep, 3(3).

41. Weiner, G. J. (2010). Rituximab: Mechanism of action. Seminars in Hematology, 47(2), 115-123.

42. Feugier, P. (2015). A review of rituximab, the first anti-CD20 monoclonal antibody used in the treatment of B non-Hodgkin's lymphomas. Future Oncology, 11(9), 1327-1342.

43. Cordero-Reyes, A. M., Youker, K. A., \& Torre-Amione, G. (2013). The role of B-cells in heart failure. Methodist DeBakey Cardiovascular Journal, 9(1), 15-19.

44. Cordero-Reyes, A. M., et al. (2016). Full Expression of Cardiomyopathy Is Partly Dependent on B-Cells: A Pathway That Involves Cytokine Activation, Immunoglobulin Deposition, and Activation of Apoptosis. Journal of the American Heart Association, 5(1).

45. Weber, M. S., et al. (2010). B-cell activation influences T-cell polarization and outcome of anti-CD20 B-cell depletion in central nervous system autoimmunity. Annals of Neurology, 68(3), 369383.

46. Yu, M., et al. (2013). TNF-alpha-secreting B cells contribute to myocardial fibrosis in dilated cardiomyopathy. Journal of Clinical Immunology, 33(5), 1002-1008.

47. Zouggari, Y., et al. (2013). B lymphocytes trigger monocyte mobilization and impair heart function after acute myocardial infarction. Nature Medicine, 19(10), 1273-1280.

48. Touma, Z., et al. (2008). Successful treatment of cardiac involvement in dermatomyositis with rituximab. Joint, Bone, Spine, 75(3), 334-337.

49. Wessels, J. A., Huizinga, T. W., \& Guchelaar, H. J. (2008). Recent insights in the pharmacological actions of methotrexate in the treatment of rheumatoid arthritis. Rheumatology (Oxford), 47(3), 249-255.

50. Choi, H. K., et al. (2002). Methotrexate and mortality in patients with rheumatoid arthritis: A prospective study. Lancet, 359(9313), 1173-1177.

51. Ridker, P. M., et al. (2019). Low-dose methotrexate for the prevention of atherosclerotic events. The New England Journal of Medicine, 380(8), 752-762.

52. Mukherjee, S., \& Mukherjee, U. (2009). A comprehensive review of immunosuppression used for liver transplantation. $J$ Transplant, 2009, 701464.

53. Krakauer, T., et al. (2010). Rapamycin protects mice from staphylococcal enterotoxin B-induced toxic shock and blocks cytokine release in vitro and in vivo. Antimicrobial Agents and Chemotherapy, 54(3), 1125-1131.

54. Lemos, P. A., \& Bienert, I. (2013). The Supralimus sirolimuseluting stent. Expert Review of Medical Devices, 10(3), 295-300.

55. Hausleiter, J., et al. (2004). Randomized, double-blind, placebocontrolled trial of oral sirolimus for restenosis prevention in patients with in-stent restenosis: The oral Sirolimus to inhibit recurrent in-stent stenosis (OSIRIS) trial. Circulation, 110(7), 790-795.

56. Xu, L., \& Brink, M. (2016). mTOR, cardiomyocytes and inflammation in cardiac hypertrophy. Biochimica et Biophysica Acta, 1863(7 Pt B), 1894-1903.

57. Kushwaha, S., \& Xu, X. (2012). Target of rapamycin (TOR)based therapy for cardiomyopathy: Evidence from zebrafish and human studies. Trends in Cardiovascular Medicine, 22(2), 39-43.

58. Shibata, K., Endo, T., \& Kuribayashi, Y. (2019). Computational drug-repositioning approach identifying Sirolimus as a potential therapeutic option for inflammatory dilated cardiomyopathy. Drug Res (Stuttg), 69(10), 565-571. 
59. Chen, Z., et al. (2014). LY294002 and rapamycin promote coxsackievirus-induced cytopathic effect and apoptosis via inhibition of PI3K/AKT/mTOR signaling pathway. Molecular and Cellular Biochemistry, 385(1-2), 169-177.

60. Nimmerjahn, F., \& Ravetch, J. V. (2008). Anti-inflammatory actions of intravenous immunoglobulin. Annual Review of Immunology, 26, 513-533.

61. Anthony, R. M., et al. (2008). Recapitulation of IVIG antiinflammatory activity with a recombinant $\mathrm{IgG}$ fc. Science, 320(5874), 373-376.

62. Gronwall, C., \& Silverman, G. J. (2014). Natural IgM: Beneficial autoantibodies for the control of inflammatory and autoimmune disease. Journal of Clinical Immunology, 34(Suppl 1), S12-S21.

63. Bayry, J., Mouthon, L., \& Kaveri, S. V. (2012). Intravenous immunoglobulin expands regulatory $\mathrm{T}$ cells in autoimmune rheumatic disease. The Journal of Rheumatology, 39(2), 450-451.

64. Rossi, F., Dietrich, G., \& Kazatchkine, M. D. (1989). Antiidiotypes against autoantibodies in normal immunoglobulins: Evidence for network regulation of human autoimmune responses. Immunological Reviews, 110, 135-149.

65. Hampe, C. S. (2012). Protective role of anti-idiotypic antibodies in autoimmunity-lessons for type 1 diabetes. Autoimmunity, 45(4), 320-331.

66. Kazatchkine, M. D., \& Kaveri, S. V. (2001). Immunomodulation of autoimmune and inflammatory diseases with intravenous immune globulin. The New England Journal of Medicine, 345(10), 747-755.

67. McNamara, D. M., et al. (2001). Controlled trial of intravenous immune globulin in recent-onset dilated cardiomyopathy. Circulation, 103(18), 2254-2259.

68. Yen, M. H., et al. (2015). Effect of intravenous immunoglobulin for neonates with severe enteroviral infections with emphasis on the timing of administration. Journal of Clinical Virology, 64, 92 96.

69. Pankuweit, S., \& Maisch, B. The heart in viral infections(2010). Internist (Berl), 51(7), 836-843.

70. Maisch, B., et al. (2004). Treatment of inflammatory dilated cardiomyopathy and (peri)myocarditis with immunosuppression and i.v. immunoglobulins. Herz, 29(6), 624-636.

71. Dennert, R., et al. (2010). Intravenous immunoglobulin therapy for patients with idiopathic cardiomyopathy and endomyocardial biopsy-proven high PVB19 viral load. Antiviral Therapy, 15(2), 193-201.

72. Trimpert, C., et al. (2010). Immunoadsorption in dilated cardiomyopathy: Long-term reduction of cardiodepressant antibodies. European Journal of Clinical Investigation, 40(8), 685-691.

73. Selbing, A., et al. (1995). Parvovirus B19 infection during pregnancy treated with high-dose intravenous gammaglobulin. Lancet, 345(8950), 660-661.

74. Markowitz, C. E. (2007). Interferon-beta: Mechanism of action and dosing issues. Neurology, 68(24 Suppl 4), S8-S11.

75. Samuel, C. E. (2001). Antiviral actions of interferons. Clinical Microbiology Reviews, 14(4), 778-809 table of contents.

76. Schultheiss, H. P., et al. (2016). Betaferon in chronic viral cardiomyopathy (BICC) trial: Effects of interferon-beta treatment in patients with chronic viral cardiomyopathy. Clinical Research in Cardiology, 105(9), 763-773.

77. Kuhl, U., et al. (2003). Interferon-beta treatment eliminates cardiotropic viruses and improves left ventricular function in patients with myocardial persistence of viral genomes and left ventricular dysfunction. Circulation, 107(22), 2793-2798.

78. Felix, S. B., et al. (2000). Hemodynamic effects of immunoadsorption and subsequent immunoglobulin substitution in dilated cardiomyopathy: Three-month results from a randomized study. Journal of the American College of Cardiology, 35(6), $1590-1598$.
79. Ameling, S., et al. (2016). Changes of myocardial gene expression and protein composition in patients with dilated cardiomyopathy after immunoadsorption with subsequent immunoglobulin substitution. Basic Research in Cardiology, 111(5), 53.

80. Bhardwaj, G., et al. (2017). Endomyocardial proteomic signature corresponding to the response of patients with dilated cardiomyopathy to immunoadsorption therapy. Journal of Proteomics, 150, 121-129.

81. Dungen, H. D., et al. (2020). Beta1-adrenoreceptor autoantibodies in heart failure: Physiology and therapeutic implications. Circulation. Heart Failure, 13(1), e006155.

82. Cao, Y., Xu, W., \& Xiong, S. (2013). Adoptive transfer of regulatory $\mathrm{T}$ cells protects against coxsackievirus B3-induced cardiac fibrosis. PLoS One, 8(9), e74955.

83. Tang, H., et al. (2010). Low responder T cell susceptibility to the suppressive function of regulatory $\mathrm{T}$ cells in patients with dilated cardiomyopathy. Heart, 96(10), 765-771.

84. Chen, P., et al. (2012). Susceptibility to autoimmune myocarditis is associated with intrinsic differences in $\mathrm{CD} 4(+) \mathrm{T}$ cells. Clinical and Experimental Immunology, 169(2), 79-88.

85. Li, J., et al. (2010). The Treg/Th17 imbalance in patients with idiopathic dilated cardiomyopathy. Scandinavian Journal of Immunology, 71(4), 298-303.

86. Shi, Y., et al. (2010). Regulatory T cells protect mice against coxsackievirus-induced myocarditis through the transforming growth factor beta-coxsackie-adenovirus receptor pathway. Circulation, 121(24), 2624-2634.

87. Keating, A. (2006). Mesenchymal stromal cells. Current Opinion in Hematology, 13(6), 419-425.

88. Ren, G., et al. (2008). Mesenchymal stem cell-mediated immunosuppression occurs via concerted action of chemokines and nitric oxide. Cell Stem Cell, 2(2), 141-150.

89. Van Linthout, S., et al. (2011). Mesenchymal stem cells improve murine acute coxsackievirus B3-induced myocarditis. European Heart Journal, 32(17), 2168-2178.

90. Savvatis, K., et al. (2012). Mesenchymal stromal cells but not cardiac fibroblasts exert beneficial systemic immunomodulatory effects in experimental myocarditis. PLoS One, 7(7), e41047.

91. Miteva, K., et al. (2018). Mesenchymal stromal cells inhibit NLRP3 inflammasome activation in a model of Coxsackievirus B3-induced inflammatory cardiomyopathy. Scientific Reports, 8(1), 2820 .

92. Miteva, K., et al. (2013). Immunomodulatory effects of mesenchymal stromal cells revisited in the context of inflammatory cardiomyopathy. Stem Cells International, 2013, 353097.

93. Miteva, K., et al. (2016). Human endomyocardial biopsy specimen-derived stromal cells modulate angiotensin II-induced cardiac remodeling. Stem Cells Translational Medicine, 5(12), $1707-1718$

94. Miteva, K., et al. (2011). Human cardiac-derived adherent proliferating cells reduce murine acute coxsackievirus B3-induced myocarditis. PLoS One, 6(12), e28513.

95. Mishima, K., \& Irie, K. (2020). Central effect of components of Cannabis: Utility and risk. Yakugaku Zasshi, 140(2), 193-204.

96. Mukhopadhyay, P., et al. (2011). Cannabidiol protects against hepatic ischemia/reperfusion injury by attenuating inflammatory signaling and response, oxidative/nitrative stress, and cell death. Free Radical Biology \& Medicine, 50(10), 1368-1381.

97. Hao, E., et al. (2015). Cannabidiol protects against doxorubicininduced cardiomyopathy by modulating mitochondrial function and biogenesis. Molecular Medicine, 21, 38-45.

98. Lee, W. S., et al. (2016). Cannabidiol limits T cell-mediated chronic autoimmune myocarditis: Implications to autoimmune disorders and organ transplantation. Molecular Medicine, 22, 136-146. 
99. McMurray, J. J., et al. (2012). ESC guidelines for the diagnosis and treatment of acute and chronic heart failure 2012: The task force for the diagnosis and treatment of acute and chronic heart failure 2012 of the European Society of Cardiology. Developed in collaboration with the heart failure association (HFA) of the ESC. European Journal of Heart Failure, 14(8), 803-869.

100. Martinez-Gonzalez, J., \& Badimon, L. (2007). Mechanisms underlying the cardiovascular effects of COX-inhibition: Benefits and risks. Current Pharmaceutical Design, 13(22), 2215-2227.

101. Martinon, F., et al. (2006). Gout-associated uric acid crystals activate the NALP3 inflammasome. Nature, 440(7081), 237-241.

102. Leung, Y. Y., Yao Hui, L. L., \& Kraus, V. B. (2015). Colchicineupdate on mechanisms of action and therapeutic uses. Seminars in Arthritis and Rheumatism, 45(3), 341-350.

103. Dalbeth, N., Lauterio, T. J., \& Wolfe, H. R. (2014). Mechanism of action of colchicine in the treatment of gout. Clinical Therapeutics, 36(10), 1465-1479.

104. Zhou, W., et al. (2018). NLRP3: A novel mediator in cardiovascular disease. Journal of Immunology Research, 2018, 5702103.

105. Tschope, C., et al. (2017). NOD2 (nucleotide-binding oligomerization domain 2) Is a major pathogenic mediator of coxsackievirus B3-induced myocarditis. Circulation. Heart Failure, 10(9).

106. Toldo, S., et al. (2014). Formation of the inflammasome in acute myocarditis. International Journal of Cardiology, 171(3), e119e121.

107. Van Linthout, S., et al. (2018). P2845Colchicine reduces NLRP3 inflammasome activity in murine coxsackievirus B3-induced myocarditis. European Heart Journal, 39(suppl_1).

108. Deftereos, S., et al. (2013). Colchicine and the heart: Pushing the envelope. Journal of the American College of Cardiology, 62(20), $1817-1825$.

109. Imazio, M., et al. (2013). A randomized trial of colchicine for acute pericarditis. The New England Journal of Medicine, 369(16), 1522-1528.

110. Imazio, M., et al. (2011). Colchicine for recurrent pericarditis (CORP): A randomized trial. Annals of Internal Medicine, 155(7), 409-414.

111. Imazio, M., et al. (2005). Colchicine in addition to conventional therapy for acute pericarditis: Results of the COlchicine for acute PEricarditis (COPE) trial. Circulation, 112(13), 2012-2016.

112. Imazio, M., et al. (2011). Colchicine reduces postoperative atrial fibrillation: Results of the colchicine for the prevention of the postpericardiotomy syndrome (COPPS) atrial fibrillation substudy. Circulation, 124(21), 2290-2295.

113. Adler, Y., et al. (2015). 2015 ESC guidelines for the diagnosis and management of pericardial diseases: The task force for the diagnosis and management of pericardial diseases of the European Society of Cardiology (ESC)endorsed by: The European Association for Cardio-Thoracic Surgery (EACTS). European Heart Journal, 36(42), 2921-2964.

114. Brucato, A., et al. (2008). Recurrent pericarditis: Infectious or autoimmune? Autoimmunity Reviews, 8(1), 44-47.

115. He, Y., Hara, H., \& Nunez, G. (2016). Mechanism and regulation of NLRP3 inflammasome activation. Trends in Biochemical Sciences, 41(12), 1012-1021.

116. Wree, A., et al. (2014). NLRP3 inflammasome activation is required for fibrosis development in NAFLD. Journal of Molecular Medicine (Berlin, Germany), 92(10), 1069-1082.

117. Sutterwala, F. S., Haasken, S., \& Cassel, S. L. (2014). Mechanism of NLRP3 inflammasome activation. Annals of the New York Academy of Sciences, 1319, 82-95.

118. Rose, N. R., \& Hill, S. L. (1996). Autoimmune myocarditis. International Journal of Cardiology, 54(2), 171-175.
119. Kraft, L., et al. (2019). Blocking the IL-1beta signalling pathway prevents chronic viral myocarditis and cardiac remodeling. Basic Research in Cardiology, 114(2), 11.

120. Furst, D. E., et al. (2006). Updated consensus statement on biological agents for the treatment of rheumatic diseases, 2006. Annals of the Rheumatic Diseases, 65(Suppl 3), iii2-iii15.

121. Ikonomidis, I., et al. (2008). Inhibition of interleukin-1 by anakinra improves vascular and left ventricular function in patients with rheumatoid arthritis. Circulation, 117(20), 2662-2669.

122. Abbate, A., et al. (2013). Effects of interleukin-1 blockade with anakinra on adverse cardiac remodeling and heart failure after acute myocardial infarction [from the Virginia Commonwealth University-Anakinra remodeling trial (2) (VCU-ART2) pilot study]. The American Journal of Cardiology, 111(10), 13941400.

123. Van Tassell, B. W., et al. (2016). Interleukin-1 blockade in acute decompensated heart failure: A randomized, double-blinded, Placebo-Controlled Pilot Study. Journal of Cardiovascular Pharmacology, 67(6), 544-551.

124. Van Tassell, B. W., et al. (2014). Effects of interleukin-1 blockade with anakinra on aerobic exercise capacity in patients with heart failure and preserved ejection fraction (from the D-HART pilot study). The American Journal of Cardiology, 113(2), 321-327.

125. Brucato, A., et al. (2016). Effect of Anakinra on recurrent pericarditis among patients with colchicine resistance and corticosteroid dependence: The AIRTRIP randomized clinical trial. JAMA, 316(18), 1906-1912.

126. Cavalli, G., et al. (2017). Interleukin-1 receptor blockade rescues myocarditis-associated end-stage heart failure. Frontiers in Immunology, 8, 131.

127. Ridker, P. M., et al. (2017). Antiinflammatory therapy with canakinumab for atherosclerotic disease. The New England Journal of Medicine, 377(12), 1119-1131.

128. Bianchi, M. E. (2007). DAMPs, PAMPs and alarmins: All we need to know about danger. Journal of Leukocyte Biology, $81(1), 1-5$.

129. Loukili, N., et al. (2010). Oxidants positively or negatively regulate nuclear factor kappaB in a context-dependent manner. The Journal of Biological Chemistry, 285(21), 15746-15752.

130. Simard, J. C., et al. (2013). S100A8 and S100A9 induce cytokine expression and regulate the NLRP3 inflammasome via ROSdependent activation of NF-kappaB(1.). PLoS One, 8(8), e72138.

131. Bauernfeind, F. G., et al. (2009). Cutting edge: NF-kappaB activating pattern recognition and cytokine receptors license NLRP3 inflammasome activation by regulating NLRP3 expression. Journal of Immunology, 183(2), 787-791.

132. Cotoi, O. S., et al. (2014). Plasma S100A8/A9 correlates with blood neutrophil counts, traditional risk factors, and cardiovascular disease in middle-aged healthy individuals. Arteriosclerosis, Thrombosis, and Vascular Biology, 34(1), 202-210.

133. Muller, I., et al. (2017). CX3CR1 knockout aggravates coxsackievirus B3-induced myocarditis. PLoS One, 12(8), e0182643.

134. Muller, I., et al. (2017). Pathogenic role of the damage-associated molecular patterns S100A8 and S100A9 in coxsackievirus B3induced myocarditis. Circulation. Heart Failure, 10(11).

135. Bjork, P., et al. (2009). Identification of human S100A9 as a novel target for treatment of autoimmune disease via binding to quinoline-3-carboxamides. PLoS Biology, 7(4), e97.

136. Tschope, C., et al. (2019). Mechanical unloading by fulminant myocarditis: LV-IMPELLA, ECMELLA, BI-PELLA, and PROPELLA concepts. Journal of Cardiovascular Translational Research, 12(2), 116-123. 
137. Van Linthout, S., \& Tschope, C. (2017). Inflammation - cause or consequence of heart failure or both? Current Heart Failure Reports, 14(4), 251-265.

138. Usher, M. G., et al. (2010). Myeloid mineralocorticoid receptor controls macrophage polarization and cardiovascular hypertrophy and remodeling in mice. The Journal of Clinical Investigation, 120(9), 3350-3364.

139. Wong, V. W., et al. (2011). Mechanical force prolongs acute inflammation via T-cell-dependent pathways during scar formation. The FASEB Journal, 25(12), 4498-4510.

140. McWhorter, F. Y., Davis, C. T., \& Liu, W. F. (2015). Physical and mechanical regulation of macrophage phenotype and function. Cellular and Molecular Life Sciences, 72(7), 1303-1316.

141. Spillmann, F., et al. (2019). Mode-of-action of the PROPELLA concept in fulminant myocarditis. European Heart Journal, 40(26), 2164-2169.

142. Cannata, A., et al. (2019). Myocarditis evolving in cardiomyopathy: When genetics and offending causes work together. European Heart Journal Supplements: Journal of the European Society of Cardiology, 21(Suppl B), B90-B95.
143. Heymans, S., et al. (2016). The quest for new approaches in myocarditis and inflammatory cardiomyopathy. Journal of the American College of Cardiology, 68(21), 2348-2364.

144. Massilamany, C., et al. (2014). Relevance of molecular mimicry in the mediation of infectious myocarditis. Journal of Cardiovascular Translational Research, 7(2), 165-171.

145. Tai, N., et al. (2016). Microbial antigen mimics activate diabetogenic CD8 T cells in NOD mice. The Journal of Experimental Medicine, 213(10), 2129-2146.

146. Gil-Cruz, C., et al. (2019). Microbiota-derived peptide mimics drive lethal inflammatory cardiomyopathy. Science, 366(6467), 881-886.

147. Buttgereit, F., et al. (2002). Standardised nomenclature for glucocorticoid dosages and glucocorticoid treatment regimens: Current questions and tentative answers in rheumatology. Annals of the Rheumatic Diseases, 61(8), 718-722.

Publisher's Note Springer Nature remains neutral with regard to jurisdictional claims in published maps and institutional affiliations. 